\title{
Immunotherapy Foreword 2018
}

\author{
Mike Gregg*,1 \\ ${ }^{1}$ Future Science Group, Unitec House, 2 Albert Place, London N31QB, UK \\ *Author for correspondence: m.gregg@futuremedicine.com
}

To all of our readers, the Future Medicine editorial office would like to thank you for your continued readership over 2018 and I hope you have a fantastic Christmas and New Year. I would also like to thank our esteemed editorial board, peer reviewers and contributing authors for their continued support. We very much look forward to working with you all in 2019 and seeing the journal continue to progress. In this foreword, we will present some highlights from the last 12 months in addition to looking forward to the year ahead.

First draft submitted: 19 November 2018; Published online: 23 November 2018

Keywords: asthma $\bullet$ cancer $\bullet$ checkpoint inhibitors $\bullet$ immunotherapy $\bullet$ JAK1 and JAK2 inhibitors $\bullet$ tumor

\section{Content highlights}

Immunotherapy has had another great year in 2018. The journal published a large number of superb articles, comprising of a broad spread of content representing the diverse field of immunotherapy.

Immunotherapy's top read article of 2018 (as of October 2018) was a review entitled 'Ruxolitinib for the treatment of patients with steroid-refractory GVHD: an introduction to the REACH trials' written by Madan Jagasia et al. [1]. This review outlines the potential of JAK1 and JAK2 inhibitors such as ruxolitinib in preventing graft-versus-host-disease, which affects patients undergoing allogeneic hematopoietic stem cell transplantation. The ever-expanding field of GVHD treatment has opened new avenues in our understanding of the disease and we encourage any submissions exploring this topic in the future.

Our second-most-read article was an editorial that evaluated the effect that combining immunotherapy checkpoint inhibitors PD1, PD-L1 and CTLA-4 have on mesothelioma [2]. Written by Monica Chatwal et al., this article concluded that this method of treatment is showing promise but further study is needed. Another article that proved very popular with our readers was an intriguing clinical trial evaluation entitled 'Tralokinumab for the treatment of severe, uncontrolled asthma: the ATMOSPHERE clinical development program' [3]. This paper, written by Reynold A Panettieri et al. investigates the long-term safety and effectiveness of using tralokinumab in the treatment of severe cases of asthma. Finally of note is a review authored by Stephanie K Dougan and Michael Dougan entitled 'Regulation of innate and adaptive antitumor immunity by IAP antagonists' [4]. An article that also attracted a great deal of attention, it looked at the potential of using inhibitors of apoptosis (IAP) protein family in checkpoint blockade for cancers that are unreceptive to CTLA-4, PD-1 and PD-L1. The study found that these inhibitors were effective in enhancing tumor cell killing and are set to become an important immunotherapy treatment in the future.

\section{Readership demographics}

It is always interesting to consider the impact of our content, and as the journal grows, our readership demographic also changes. This year the USA came top again for readership of Immunotherapy with 37\%, with Asia (29\%) and Europe (27\%) following closely (Figure 1). While our content continues to have an impact across Europe and the West, we expect to see continued growth in emerging scientific communities and hope to continue our work toward reaching a truly global audience.

\section{Top articles on Altmetric}

We have been working with Altmetric since 2015 to help visualize where the articles we publish are having online impact. A special acknowledgement goes out to this year's top three articles, which all scored in the top $25 \%$ of all research outputs scored by Altmetric. 

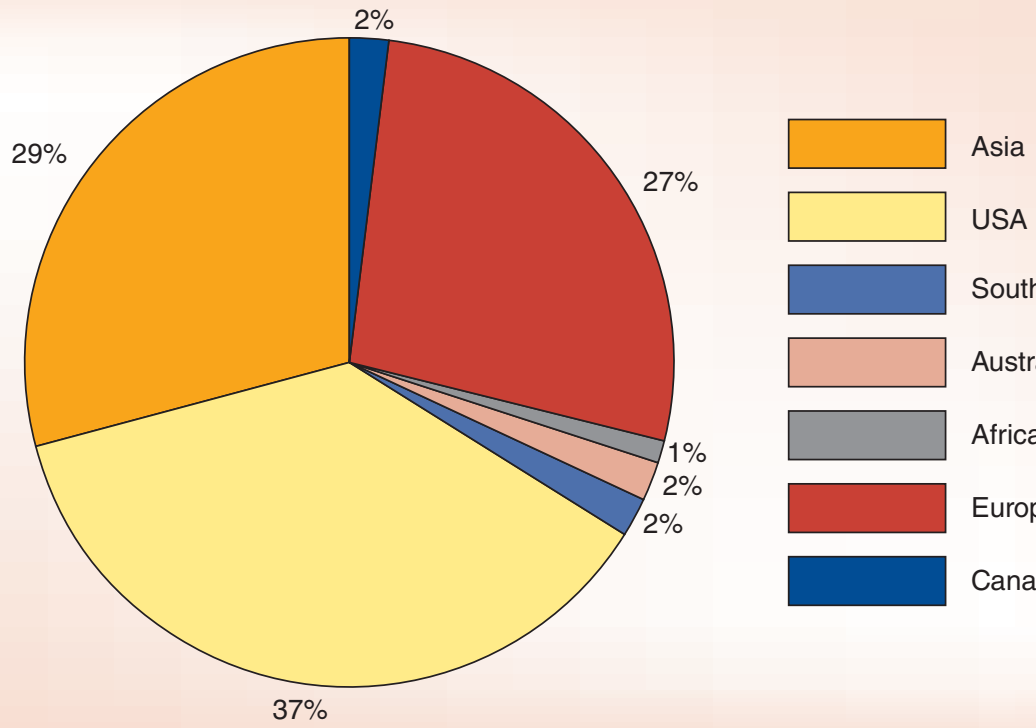

South America

Australasia

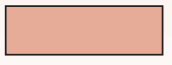

Africa

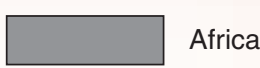

Europe

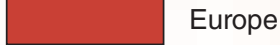

Canada

Figure 1. Journal of Immunotherapy readership demographic 2018.

- First was a research article entitled 'Long-term effect of monophosphoryl lipid A adjuvanted specific immunotherapy in patients with grass pollen allergy' written by Stefan Zielan et al. This article did incredibly well and was in the top 5\% of all research outputs scored by Altmetric this year [5];

- Second, was the article entitled 'A network meta-analysis of the $\mathrm{PD}(\mathrm{L})-1$ inhibitors in the salvage treatment of urothelial bladder cancer' [6];

- In third place was the very topical clinical trial evaluation entitled 'Tralokinumab for the treatment of severe, uncontrolled asthma: the ATMOSPHERE clinical development program', as discussed above [3].

\section{Social media}

Immunotherapy remains active across social media and you can follow our latest updates on Twitter (@FsgIMT) and LinkedIn [7]. Across these platforms, we discuss the most recent research in the field of immunotherapy, and the latest news, journal highlights and newly published articles. Through these mediums, we continue to enjoy engaging with our readers and experts in the field.

\section{Conclusions}

As always, we welcome any feedback the immunotherapy community wishes to share, whether that be key topics that you feel should be covered or where you see the field going in the future. We welcome unsolicited research, review and opinion article proposals and would be delighted to hear from you if you are interested in submitting to the journal. In particular, we are looking for submissions surrounding key areas such as:

- Cancer immunotherapy, including checkpoint blockade, cancer vaccines, adoptive T cell therapy, monoclonal antibodies and cytokines;

- Combined chemo/radio immunotherapy in cancer treatment;

- Biomarkers for personalized cancer immunotherapy;

- Allergen immunotherapy, including sublingual, subcutaneous and oral immunotherapy;

- Restorative immunotherapy for HIV and other immunocompromised patients;

- Suppressive immunotherapy for autoimmunity diseases and transplantation;

- Basic science of immunotherapeutic approaches;

- Drug-immune system interactions;

- Drug delivery systems, drug combinations and drug-drug interactions;

- Summaries evaluating newly approved immunotherapeutic agents;

- Newly identified immune targets of drugs; 
- Results of recent findings and clinical trials relevant to the field of immunotherapy;

- Post marketing research, including adverse events, drug safety, pharmacoeconomics, cost-benefit issues, realworld evidence and outcomes research.

We greatly look forward to collaborating with you next year and hope to see Immunotherapy continue to grow and impact the scientific community in 2019.

\section{Financial \& competing interest's disclosure}

Mike Gregg is an employee of Future Medicine Ltd. The author has no other relevant affiliations or financial involvement with any organization or entity with a financial interest in or financial conflict with the subject matter or materials discussed in the manuscript apart from those disclosed.

No writing assistance was utilized in the production of this manuscript.

\section{References}

1. Jagasia M, Zeiser R, Arbushites M, Delaite P, Gadbaw B, von Bubnoff N. Ruxolitinib for the treatment of patients with steroid-refractory GVHD: an introduction to the REACH trials. Immunotherapy 10(5), 391-402 (2018).

2. Chatwal SM, Tanvetyanon T. Malignant mesothelioma clinical trial combines immunotherapy drugs. Immunotherapy 10(5), 341-344 (2018).

3. Panettieri AR, Wang M, Braddock M, Bowen K, Colice G. Tralokinumab for the treatment of severe, uncontrolled asthma: the ATMOSPHERE clinical development program. Immunotherapy 10(6), 473-490 (2018).

4. Dougan SK, Dougan M. Regulation of innate and adaptive antitumor immunity by IAP antagonists. Immunotherapy. 10(9), 787-796 (2018).

5. Zielen S, Gabrielpillai J, Herrmann E, Schulze J, Schubert R, Rosewich M. Long-term effect of monophosphoryl lipid A adjuvanted specific immunotherapy in patients with grass pollen allergy. Immunotherapy 10(7), 529-536 (2018).

6. Rassy EE, Bakouny Z, Aoun F, Haddad FG, Sleilaty G, Assi T, Kattan J. A network meta-analysis of the PD(L)-1 inhibitors in the salvage treatment of urothelial bladder cancer. Immunotherapy 10(8), 657-663 (2018).

7. Immunotherapy's Linkedin page: www.linkedin.com/groups/3713578/ 
